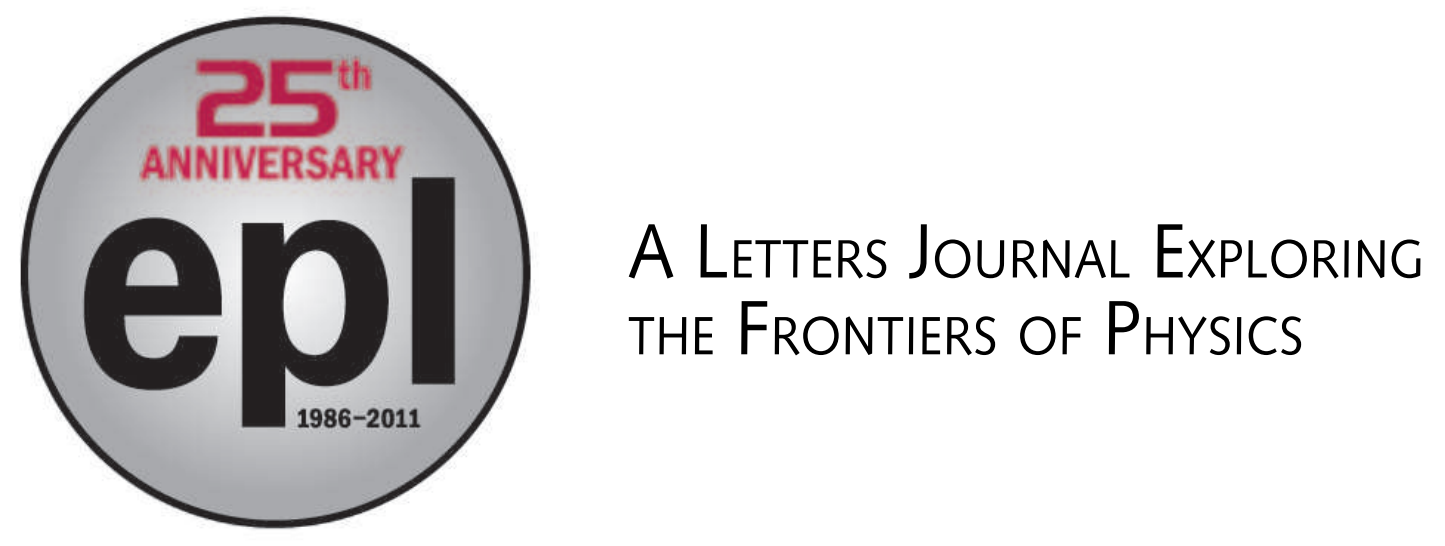

\title{
OFFPRINT
}

\section{Fission diamond detectors for fast-neutron ToF spectroscopy}

A. Pietropaolo, C. Andreani, M. Rebai, L. Giacomelli, G. Gorini, E. Perelli Cippo, M. Tardocchi, A. Fazzi, G. Verona Rinati, C. Verona, Marco Marinelli, E. Milani, C. D. Frost and E. M. SCHOONEVELD

EPL, 94 (2011) 62001

Please visit the new website www.epljournal.org 


\section{A LETTERS JOURNAL EXPLORING THE FRONTIERS OF PHYSICS \\ The Editorial Board invites you to submit your letters to EPL www.epl journal.org}

\section{Six good reasons to publish with EPL}

We want to work with you to help gain recognition for your high-quality work through worldwide visibility and high citations. As an EPL author, you will benefit from:

1 Quality - The 40+ Co-Editors, who are experts in their fields, oversee the entire peer-review process, from selection of the referees to making all final acceptance decisions

2 Impact Factor - The 2009 Impact Factor increased by 31\% to 2.893; your work will be in the right place to be cited by your peers

3 Speed of processing - We aim to provide you with a quick and efficient service; the median time from acceptance to online publication is 30 days

4. High visibility - All articles are free to read for 30 days from online publication date

5 International reach - Over 2,000 institutions have access to EPL, enabling your work to be read by your peers in 100 countries

6 Open Access - Experimental and theoretical high-energy particle physics articles are currently open access at no charge to the author. All other articles are offered open access for a one-off author payment $(€ 1,000)$

Details on preparing, submitting and tracking the progress of your manuscript from submission to acceptance are available on the EPL submission website www.epletters.net

If you would like further information about our author service or EPL in general, please visit www.epljournal.org or e-mail us at info@epljournal.org 


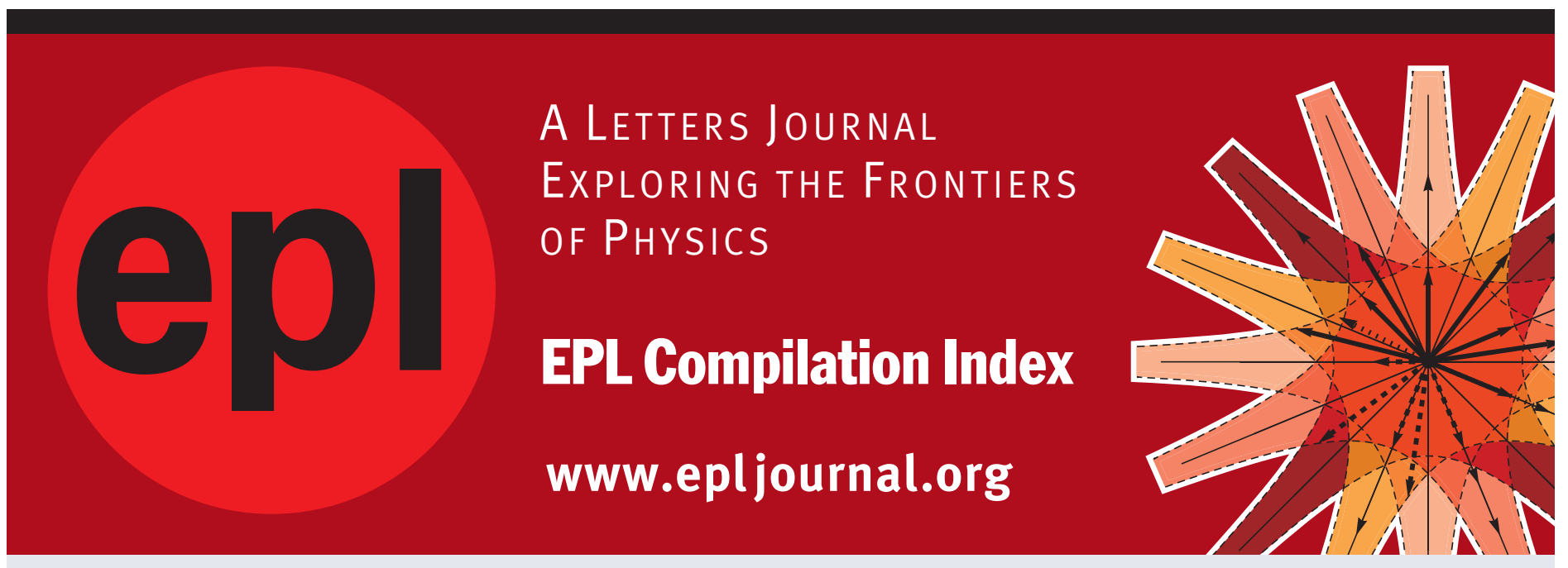

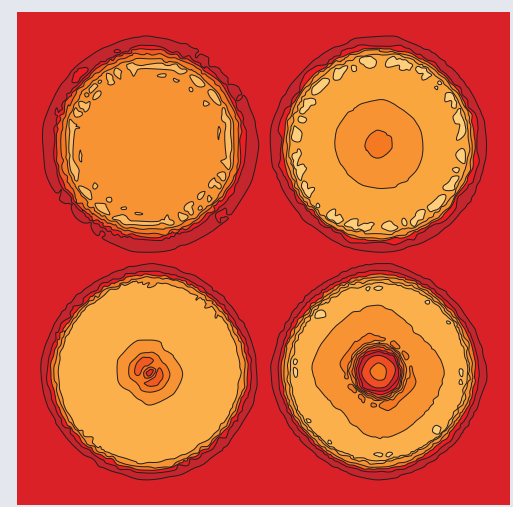

Biaxial strain on lens-shaped quantum rings of different inner radii, adapted from Zhang et al 2008 EPL 8367004.

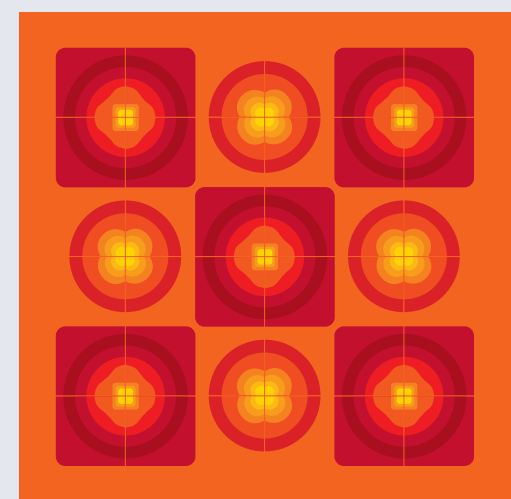

Artistic impression of electrostatic particle-particle interactions in dielectrophoresis, adapted from N Aubry and P Singh 2006 EPL 74623.

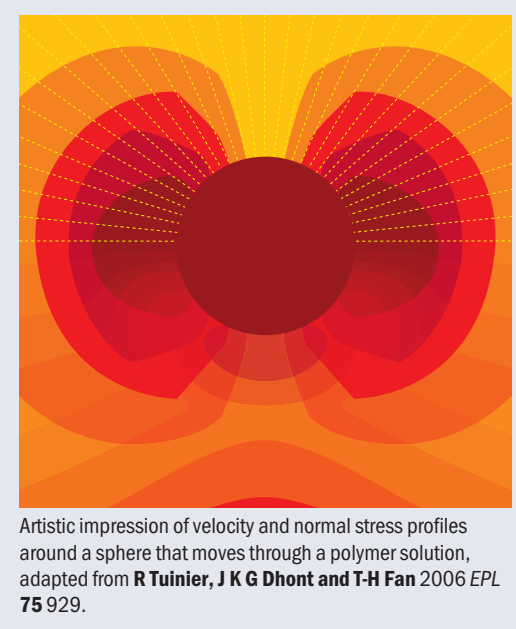

Visit the EPL website to read the latest articles published in cutting-edge fields of research from across the whole of physics.

Each compilation is led by its own Co-Editor, who is a leading scientist in that field, and who is responsible for overseeing the review process, selecting referees and making publication decisions for every manuscript.

- Graphene

- Liquid Crystals

- High Transition Temperature Superconductors

- Quantum Information Processing \& Communication

- Biological \& Soft Matter Physics

- Atomic, Molecular \& Optical Physics

- Bose-Einstein Condensates \& Ultracold Gases

- Metamaterials, Nanostructures \& Magnetic Materials

- Mathematical Methods

- Physics of Gases, Plasmas \& Electric Fields

- High Energy Nuclear Physics

If you are working on research in any of these areas, the Co-Editors would be delighted to receive your submission. Articles should be submitted via the automated manuscript system at www.epletters.net

If you would like further information about our author senvice or EPL in general, please visit www.epljournal.org or e-mail us at info@epljournal.org

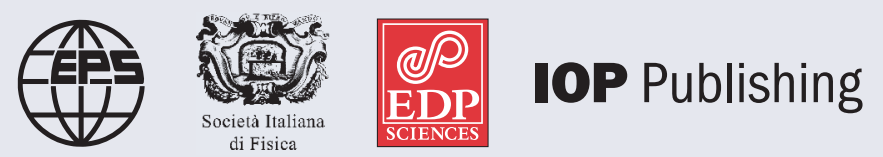

Image: Ornamental multiplication of space-time figures of temperature transformation rules (adapted from T. S. Bíró and P. Ván 2010 EPL 89 30001; artistic impression by Frédérique Swist). 


\title{
Fission diamond detectors for fast-neutron ToF spectroscopy
}

\author{
A. Pietropaolo ${ }^{1(a)}$, C. Andreani ${ }^{1}$, M. Rebai $^{2}$, L. Giacomelli ${ }^{2}$, G. Gorini ${ }^{2}$, E. Perelli Cippo $^{2}$, \\ M. Tardocchi ${ }^{3}$, A. Fazzi ${ }^{4}$, G. Verona Rinati ${ }^{5}$, C. Verona ${ }^{5}$, Marco Marinelli ${ }^{5}$, E. Milani ${ }^{5}$, \\ C. D. Frost ${ }^{6}$ and E. M. Schooneveld ${ }^{6}$ \\ ${ }^{1}$ Università degli Studi di Roma Tor Vergata, Dipartimento di Fisica and Centro NAST \\ Via della Ricerca Scientifica 1, I-00133 Roma, Italy, EU \\ ${ }^{2}$ CNISM and Dipartimento di Fisica "G. Occhialini", Università degli Studi di Milano-Bicocca - Milano, Italy, EU \\ ${ }^{3}$ CNR-IFP - Milano, Italy, EU \\ ${ }^{4}$ Department of Energy, Politecnico di Milano - Milano, Italy, EU \\ ${ }^{5}$ Dipartimento di Ingegneria Meccanica, Università degli Studi di Roma Tor Vergata - Roma, Italy, EU \\ ${ }^{6}$ STFC-ISIS Facility - Chilton Didcot, Oxfordshire, UK, EU
}

received 14 February 2011; accepted in final form 27 April 2011

published online 2 June 2011

PACS 29.30.Hs - Neutron spectroscopy

PACS 25.85.Ec - Neutron-induced fission

PACS 89.20.Bb - Industrial and technological research and development

\begin{abstract}
A novel type of fast-neutron (energy $E_{\mathrm{n}}>1 \mathrm{MeV}$ ) counter is presented. It is made of a fissionable natural-uranium foil faced to an intrinsic single-crystal diamond that detects the neutron-induced fission fragments escaping the uranium sheet. The fast response of the diamond is a key feature for its use at pulsed spallation neutron sources for applications in beam monitoring and spectrum measurements with $\mathrm{mm}$ spatial resolution. This is an important issue to be addressed in the development of beam lines dedicated to the investigation of the so-called single-event effects in electronics, such as the ChipIr instrument designed for the ISIS spallation source in the UK. Tests of the device at the ROTAX beam line at ISIS have shown its potentiality for the proposed application.
\end{abstract}

Localized neutron flux monitoring is an important issue to be addressed in high-energy neutron irradiation of microelectronics [1-3]. The determination of the crosssection of the so-called single-event effect (SEE) is related to the number of errors of a given type, registered during the irradiation run, and to the neutron fluence onto the chip $[4,5]$. Neutron detectors featuring $\mathrm{mm}^{2}$ sensible surface are desirable for this kind of applications especially at spallation neutron sources, where the intensity of fast neutrons (energy, $E_{\mathrm{n}}>1 \mathrm{MeV}$ ) is appreciable. For example, the results obtained at the ISIS spallation source on chip irradiation $[2,3]$ helped the design and future realization of the ChipIr beam line which is dedicated to this kind of applications [6]. An important requirement for this experimental irradiation station is the development of neutron counters capable of measuring the neutron fluence onto the chip, and more desirably the local spectral intensity of the neutron beam. The device should be able to exploit the pulsed nature of the spallation neutron source,

\footnotetext{
(a) E-mail: antonino.pietropaolo@roma2.infn.it
}

allowing the use of the time-of-flight (ToF) technique with fast neutrons over typical distances of $10-15 \mathrm{~m}$, available for ISIS beam lines at Target Station 2 (TS2). To this aim a device was developed that exploits the neutron-induced fission reactions in natural uranium ( ${ }^{\text {nat } U}$ ), in turn to detect the fission fragments with a single-crystal diamond detector (SDD) [7-10].

Figure 1 shows the schematic of the device, henceforth named fission diamond detector (FDD). The thickness of the fissionable ${ }^{\text {nat }} \mathrm{U}$ target is $20 \mu \mathrm{m}$, while that of the SDD is $25 \mu \mathrm{m}$. The ${ }^{n a t} U$ foil thickness allows only the fission fragments originating on the surface to escape and reach the diamond crystal where these are completely stopped. This is clear from table 1 where the ranges in uranium and diamond are listed for a typical fission fragment (rhodium) produced in ${ }^{\text {nat }} \mathrm{U}(\mathrm{n}, \mathrm{f})$ reactions.

The diamond used for the measurements presented here was grown as a small single crystal (few $\mathrm{mm}^{2}$ effective area and sub-mm thickness) by microwave chemical vapour deposition [7]. A highly conductive boron-doped diamond film of $20 \mu \mathrm{m}$ thickness is first grown epitaxially on 


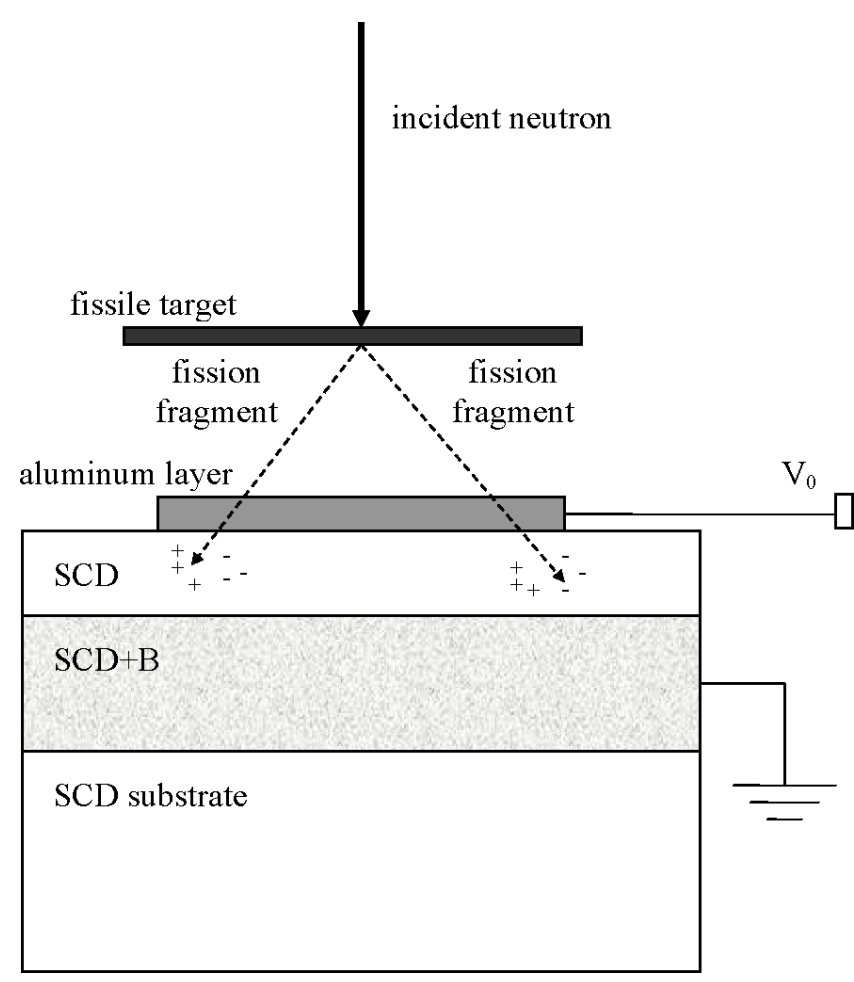

Fig. 1: Schematic of the fission diamond detector (FDD). The SCD layer is the single-crystal diamond which is the active layer of the device used to detect the fission fragments (see text for details).

Table 1: Nuclear ranges of rhodium nuclide $(A=102, Z=45)$ at three different energies in ${ }^{\text {nat }} \mathrm{U}$ and diamond.

\begin{tabular}{lccc}
\hline & $\begin{array}{c}\text { Range }(\mu \mathrm{m}) \\
\text { at } 60 \mathrm{MeV}\end{array}$ & $\begin{array}{c}\text { Range }(\mu \mathrm{m}) \\
\text { at } 90 \mathrm{MeV}\end{array}$ & $\begin{array}{c}\text { Range }(\mu \mathrm{m}) \\
\text { at } 300 \mathrm{MeV}\end{array}$ \\
\hline Uranium & 4,0 & 5.2 & 10.7 \\
Diamond & 5.8 & 7.4 & 16.7 \\
\hline
\end{tabular}

a $4 \times 4 \mathrm{~mm}^{2}$ commercial high-pressure high-temperature (HPHT) diamond substrate. This B-doped layer is used as a back contact. A thick (e.g., $25 \mu \mathrm{m})$ intrinsic diamond layer is then grown on the doped surface in a separate clean reactor, in order to avoid boron contamination of the intrinsic layer. After the growth, the intrinsic diamond layer is oxidized by isothermal annealing at $500^{\circ} \mathrm{C}$ for $1 \mathrm{~h}$ in air, in order to remove the $\mathrm{H}_{2}$ surface conductive layer. On top of the layered diamond structure, a $2.5 \mathrm{~mm}$ diameter, $100 \mathrm{~nm}$ thick aluminium layer is thermally deposited, which is used as top contact. Because of its layered geometry the SDD is only sensitive to interactions occurring within the intrinsic layer (which defines the SDD thickness). Interactions taking place in the much thicker HPHT substrate do not produce charge collected at the electrical contacts. Thus there is no need to remove the substrate, which makes the detector more robust from a mechanical point of view.

The SDD structure acts as a p-type/intrinsic/metal Schottky Barrier Diode. The aluminium contact creates a
Schottky junction with the intrinsic diamond that, in turn, is the detector layer sensitive to the ionizing radiation (drift-layer). The SDD's B-doped layer acts as a p-type layer (hole injector) and determines the unipolarity of the device under direct polarization. The device operates in reverse biased mode, with a positive voltage applied to the top metal contact and a grounded B-doped contact. The electric field in the drift layer is $E=2 \cdot 10^{4} \mathrm{~V} \mathrm{~cm}^{-1}$. Electron-hole pairs generated in the diamond will be collected at the contacts, provided that the diamond quality is such that recombination and capture (trapping) are negligible. In that case the effective charge carrier lifetime, $\tau$, is longer than the charge drift time, $\tau_{\text {drift }}$, across the detector given by

$$
\tau_{\text {drift }}=\frac{D}{\mu_{0} E}\left(1+\frac{\mu_{0} E}{v_{\text {sat }}}\right)
$$

where $D$ is the depletion layer depth, $\mu_{0}$ is the electron mobility and $v_{\text {sat }}$ is the saturation velocity. Taking into account that the detector operates in full depletion mode when the applied voltage is high $\left(V_{0} \sim 1 \mathrm{~V}_{\mu \mathrm{m}^{-1}}\right)$ and using the values $D=25 \mu \mathrm{m}, \mu_{0}=2200 \mathrm{~cm}^{2} \mathrm{~V}^{-1} \mathrm{~s}^{-1}$ and $v_{\text {sat }}=1.35 \cdot 10^{7} \mathrm{~cm} \mathrm{~s}^{-1}$ [11], eq. (1) gives $\tau_{\text {drift }} \approx 0.24 \mathrm{~ns}$.

The FDD was irradiated on the ROTAX test beam line at the ISIS spallation source. Neutrons are produced by a double bunch-structured proton beam, the bunches being about $70 \mathrm{~ns}$ wide and separated by about $300 \mathrm{~ns}$. The FDD was placed in the direct beam, exiting a methane moderator at $95 \mathrm{~K}$, at a distance of $15.5 \mathrm{~m}$ from the moderator. The neutron energy $\left(E_{\mathrm{n}}\right)$ spectrum is known to feature a peak at about $10 \mathrm{meV}$ and a $1 / E_{\mathrm{n}}$ tail in the epithermal/fast-neutron region. For each event, the electronics, setup in a biparametric configuration $[12,13]$, allowed to record the time and pulse height (i.e., the energy deposited in the diamond). It has to be stressed that the neutron-induced signals are due to fission fragments generated in the ${ }^{n a t} U$ sheet as well as to neutron inelastic reactions $[10,14]$ and neutron capture reactions, such as ${ }^{12} \mathrm{C}(\mathrm{n}, \alpha){ }^{9} \mathrm{Be}$ and ${ }^{12} \mathrm{C}(\mathrm{n}, \mathrm{n}) 3 \alpha$, in ${ }^{12} \mathrm{C}$ of SDD. The electronic chain for the data recording consisted of a custom built charge preamplifier (integration time of $500 \mathrm{~ns}$ that well matches the sampling time of the digitizer described below and gain of about $0.3 \mathrm{mV} \mathrm{fC}^{-1}$ ), a timing filter amplifier for additional shaping and gain adjustment and a N1728 CAEN Waveform Digitizer [15]. The timing filter amplifier was used to amplify the signal by a factor of about 4 , with an integration constant of $20 \mathrm{~ns}$, in order to reduce the noise and to match the $10 \mathrm{~ns}$ sampling rate of the digitizer. The electronic threshold for the acquisition of the events in the detector was set to a value corresponding to $E_{\mathrm{n}} \approx 2.5 \mathrm{MeV}$. The CAEN digitizer implements a trapezoidal filter to evaluate the pulse height from a single waveform $[15,16]$. The digitizer stores both the waveforms and biparametric data, i.e., the time of arrival of the neutron on the detector and the pulse height, in the memory of the PC. The time of flight $\left(t_{\mathrm{ToF}}\right)$ is then calculated offline as the difference between the time of interaction of the 


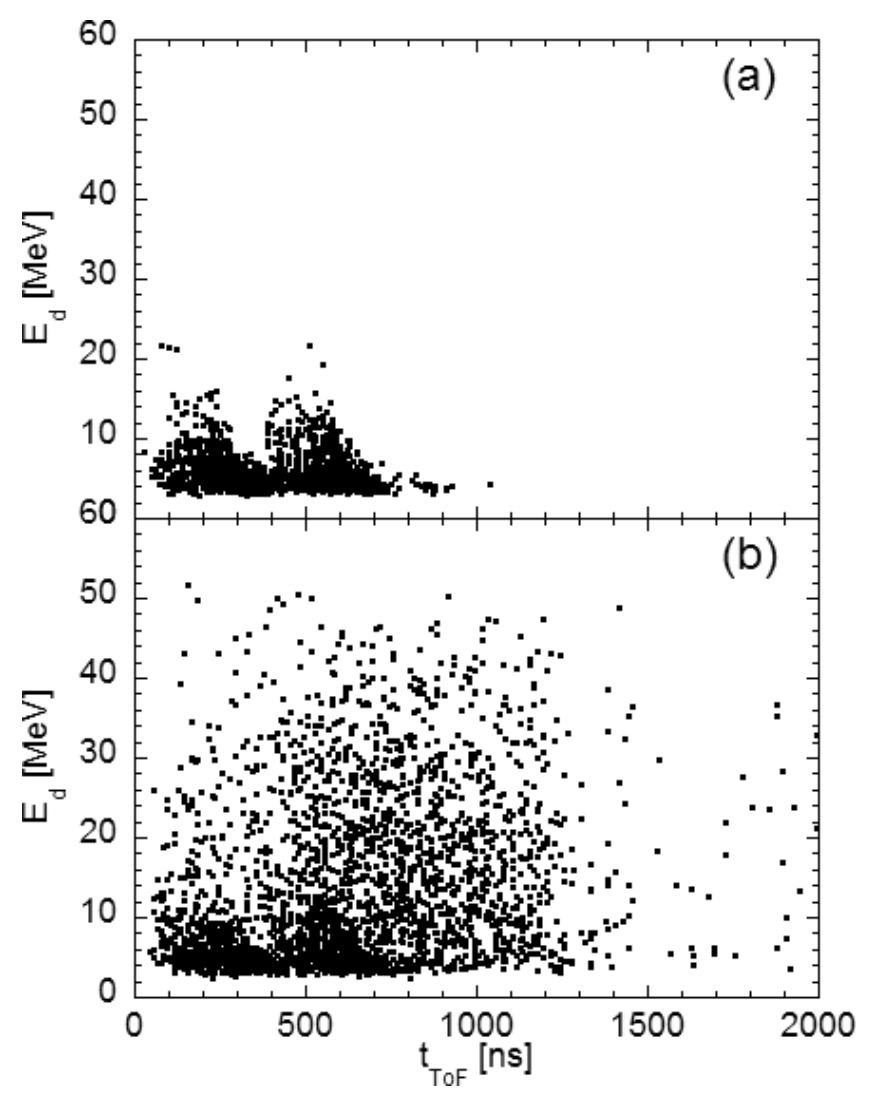

Fig. 2: Scatter plot showing the results of biparametric measurements performed with a single-crystal diamond detector without (a) and with (b) ${ }^{\text {nat }} \mathrm{U}$ fissile target foil.

neutron in the detector and the start time, i.e., the instant when the proton beam hits the spallation target.

The uranium fissionable target allows for an intrinsic energy calibration of the detector, using the $4.2 \mathrm{MeV}$ energy $\alpha$-particles produced by natural radioactivity that reach the SDD with energy $E_{\alpha} \leqslant E_{\alpha}^{\mathrm{MAX}}$ of about $4 \mathrm{MeV}$ due to the $2 \mathrm{~mm}$ air gap between the diamond and the uranium foil.

Figure 2 shows a typical biparametric spectrum of the data recorded without (a) and with (b) the fissionable ${ }^{n a t} \mathrm{U}$ target foil in front of the $25 \mu \mathrm{m}$ thick SDD. On the vertical axis the pulse height is shown in terms of the energy deposited in the detector, $E_{\mathrm{d}}$; on the horizontal axis the time of flight $t_{\mathrm{ToF}}$ (time of arrival of the pulse). Based on the events measured by the detector, and shown in fig. 2(a), we note that events due to the $\mathrm{n}-{ }^{12} \mathrm{C}$ reactions occur before $800 \mathrm{~ns}$ and deposit less energy than approximately $20 \mathrm{MeV}$ in the detector. Indeed, the energy threshold of the $\mathrm{n}^{12} \mathrm{C}$ reaction is $E^{\mathrm{TH}} \approx 6 \mathrm{MeV}$, that corresponds to a $t_{\mathrm{ToF}}$ of about $460 \mathrm{~ns}$ for a neutron produced at $t=0$ (i.e., the beginning of the first proton bunch) travelling over a flight path of $15.5 \mathrm{~m}$. Considering the proton pulse width and the time separation mentioned before, one expects that the spectrum should extend up to about $830 \mathrm{~ns}$, as can be seen in fig. 2(a). From the comparison of the two plots in fig. 2, three regions are

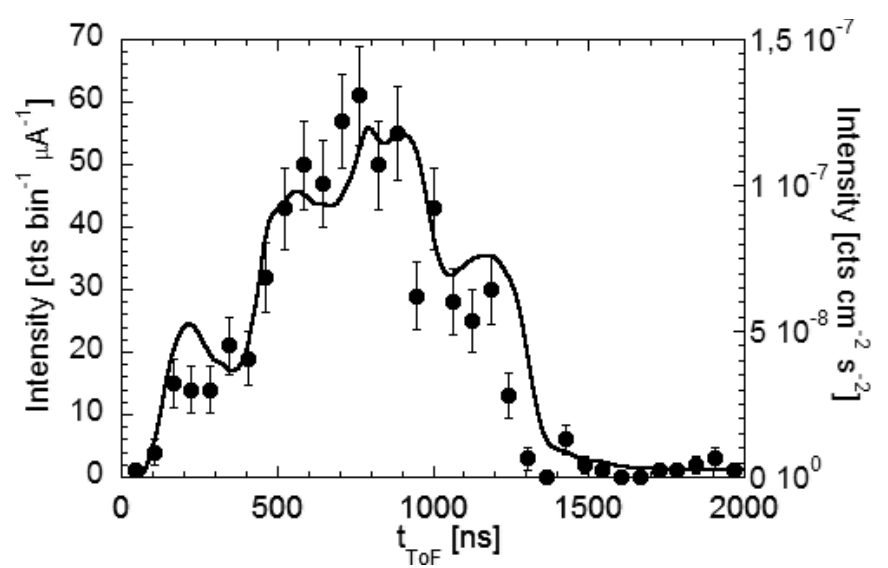

Fig. 3: Experimental ToF spectrum (dots), measured with FDD, for selected events with an energy $E_{\mathrm{d}}>20 \mathrm{MeV}$. The calculated ToF spectrum (solid line) is obtained using the fission cross-section of ${ }^{\text {nat }} \mathrm{U}$, the $E-T o F$ Jacobian and the neutron flux (see ref. [14] for details). The broadening of the spectrum, caused by the time structure of the ISIS proton bunch, has also been taken into account in these calculations.

distinguished:

1) The region with a $t_{\mathrm{ToF}}<800 \mathrm{~ns}$ and $E_{\mathrm{d}}<20 \mathrm{MeV}$, containing events due to both the $\mathrm{n}^{-12} \mathrm{C}$ reactions, as in fig. 2(a), and the fission fragments originating from the interaction of fast neutrons with ${ }^{\text {nat }} \mathrm{U}$. The fission fragments are mostly originated from ${ }^{238} \mathrm{U}$; the contribution of ${ }^{235} \mathrm{U}$ is comparatively low and can be estimated from the natural abundance of uranium (99.3\% of ${ }^{238} \mathrm{U}$ and $0.7 \%$ of $\left.{ }^{235} \mathrm{U}\right)$.

2) Events with a $t_{\mathrm{ToF}}$ between $800 \mathrm{~ns}$ and $1500 \mathrm{~ns}$ are due to fission fragments since $\mathrm{n}^{12} \mathrm{C}$ reactions are below threshold $\left(E^{\mathrm{TH}} \approx 6 \mathrm{MeV}\right)$. Comparing the energy deposited in the diamond in fig. 2(a) and in fig. 2(b) we can infer that all the events with $E_{\mathrm{d}}>20 \mathrm{MeV}$ and $t_{\mathrm{ToF}}<1500 \mathrm{~ns}$ are due to fission events mostly on ${ }^{238} \mathrm{U}$.

3) Events with a $t_{\mathrm{ToF}}>1500 \mathrm{~ns}$ are attributed to ${ }^{235} \mathrm{U}$ fission events, since the correspondent neutron energies $(800 \mathrm{keV})$ are below the energy threshold for neutron-induced fission on ${ }^{238} \mathrm{U}$.

Figure 3 (dots with statistical error bars) shows the ToF spectrum obtained by projecting the events in the biparametric spectrum of fig. 2(b) onto the $t_{\mathrm{ToF}}$ axis and adopting an offline pulse height cut for events with $E_{\mathrm{d}} \leqslant 20 \mathrm{MeV}$. As discussed before, this cut allows selecting only the events induced by the fission fragments escaping from the ${ }^{\text {nat }} \mathrm{U}$ target (see fig. 2), thus excluding those due to the direct interaction of neutrons with ${ }^{12} \mathrm{C}$ in the SDD [14]. Indeed, the biparametric data acquisition is very useful to discriminate these two contributions.

The expected ToF spectrum can be calculated by using the ${ }^{n a t} U$ fission cross-section, the Energy-ToF Jacobian 
function and the flux at the irradiation position. The latter was determined using the MCNPX Monte Carlo code $[14,17]$. The calculated ToF spectrum was then broadened by a convolution with the time structure of the ISIS proton beam (two bunches of about $70 \mathrm{~ns}$ width and separated by about $300 \mathrm{~ns})[14,18]$.

The solid line in fig. 3 (referred to the right axis scale) represents this calculation, that shows the satisfactory agreement with data (referred to the left-axis scale). This confirms the correct interpretation of the measured spectrum as due to ${ }^{238} \mathrm{U}$ fission events with little or no contribution from other processes or other types of radiation (e.g., $\gamma$-rays).

In summary, a fast-neutron counter was designed and developed using a ${ }^{\text {nat }} U$ foil as fissile target placed in front of a single-crystal diamond detector. The complete device, named Fission Diamond Detector, can reveal the fission fragments produced by fast-neutron interactions in ${ }^{\text {nat }} U$. The results obtained in the tests performed with this novel counter at the ISIS spallation source, confirm the potential application of this device for neutron beam monitoring at spallation neutron sources, justifying the further development of the detector for ChipIr applications. In fact, the small size of the device allows for localized beam monitoring, which is an important requirement for chip irradiation purposes. The accurate determination of the efficiency and response function of FDDs as a function of neutron energy will allow the application of these devices to be extended towards spectral flux characterization on $\mathrm{mm}^{2}$ scale.

$$
* * *
$$

This work was supported within the CNR-CCLRC Agreement No. 01/9001 concerning collaboration in scientific research at the spallation neutron source ISIS. The financial support of the Consiglio Nazionale delle Ricerche in this research is hereby acknowledged. One of the authors (AP) acknowledges the CNISM-CNR joint research program.

\section{REFERENCES}

[1] Ziegler J. F. et al., IBM J. Res. Dev., 40 (1996) 3.

[2] Violante M., Sterpone L., Manuzzato A., Gerardin S., Rech P., Bagatin M., Paccagnella A., Andreani C., Pietropaolo A., Cardarilli G.,
Pontarelli S. and Frost C., IEEE Trans. Nucl. Sci., 54 (2007) 1184.

[3] Andreani C., Pietropaolo A., Salsano A., Gorini G., Tardocchi M., Paccagnella A., Gerardin S., Frost C. D., Ansell S. and Platt S. P., Appl. Phys. Lett., 92 (2008) 114101.

[4] Baranov S. V., Vaselegin B. V., Osipenko P. N., Chumakov A. I. and Yanenko A. V., Russ. Microelectron., 37 (2008) 47.

[5] Juste A., Tripathi S. M. and Wijngaarden D. A., Nucl. Instrum. Methods A, 500 (2003) 371.

[6] http://www.isis.stfc.ac.uk/instruments/chipir/.

[7] Marinelli M., Milani E., Paoletti A., Tucciarone A., Verona Rinati G., Angelone M. and Pillon M., Appl. Phys. Lett., 75 (1999) 3216.

[8] Marinelli M., Milani E., Paoletti A., Tucciarone A., Verona Rinati G., Angelone M. and Pillon M., J. Appl. Phys., 89 (2001) 1430.

[9] Pietropaolo A., Verona Rinati G., Verona C., Schooneveld E. M., Angelon M. and Pillon M., Nucl. Instrum. Methods A, 610 (2009) 677.

[10] Lattanzi M. D., Angelone M., Pillon M., Almaviva S., Marinelli M., Milani E., Prestopino G., Tucciarone A., Verona C., Verona-Rinati G., Popovichev S., Monterealid R. M., Vincenti M. A., Murari A. and JET-EFDA, Fusion Eng. Des., 84 (2009) 1156.

[11] Pomorski M., Berdermann E., Ciobanu M., Martemyianov A., Moritz P., Rebisz M. and Marczewska B., Phys. Status Solidi (A), 202 (2005) 11.

[12] Tardocchi M., Pietropaolo A., Andreani C., Bracco A., D'Angelo A., Gorini G., Imberti S., Senesi R., Rhodes N. J. and Schooneveld E. M., Nucl. Instrum. Methods A, 526 (2004) 477.

[13] Tardocchi M., Gorini G., Pietropaolo A., Andreani C., Senesi R., Rhodes N. and Schooneveld E. M., Rev. Sci. Instrum., 75 (2004) 4880.

[14] Pietropaolo A., Andreani C., Rebai M., Gorini G., Giacomelli L., Perelli Cippo E., Tardocchi M., Fazzi A., Verona Rinati G., Verona C., Marco Marinelli, Milani E., Frost C. D. and Schooneveld E. M., EPL, 92 (2010) 68003.

[15] Jordanov V. T. and Knoll G. F., Nucl. Instrum. Methods A, 345 (1994) 337.

[16] CAEN Nuclear Physics, Technical information manual MOD. N1728A/B 4 CH $100 \mathrm{MHz}$ flash ADC - Manual Revision No. 5, NPO: 00118/04:N1728x.MUTx/05 (2007).

[17] http://mcnp-green.lanl.gov/.

[18] Smirnov A. N., in preparation. 